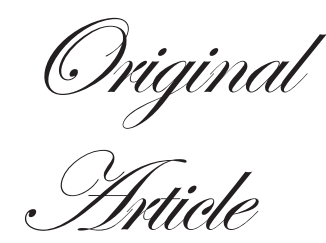

\title{
Role of endoscopic retrograde cholangiography in ruptured hepatic hydatid cyst
}

\author{
Pankaj Gupta ${ }^{1}$, Uma Debi ${ }^{1}$, Saroj Kant Sinha ${ }^{1}$, Kaushal Kishor \\ Prasad $^{2}$
}

\begin{abstract}
Department of Gastroenterology, Radiology Section ${ }^{1}$ Pathology Section ${ }^{2}$ Post Graduate Institute of Medical Education and Research,

Chandigarh, India-160012

Correspondence

Dr. Uma Debi

Email:debiuma@yahoo.co.in

Aim: To report endoscopic retrograde cholangiopancreatography (ERCP) findings of intrabiliary hydatid cyst rupture

Material and methods: A retrospective study of cases with intrabiliary rupture of hydatid cyst was conducted. A total of 4 cases (all female with a mean age of 32 years) were included. ERCP and ultrasound data were evaluated. Assessment involved number, location, size, internal and wall characteristics of cyst; intrahepatic biliary radicle dilatation (IHBRD); common bile duct (CBD) dilatation and evidence of extrabiliary rupture on ultrasound. ERCP evaluation included IHBRD, CBD dilatation, communication between cysts and intrahepatic biliary radicles (IHBR) and filling defects within the IHBR and CBD. Treatment details were also obtained. Results: A total of 5 cysts were evaluated in 4 patients. Left lobe cysts were most common $(n=4)$. Segment II/III were the most commonly affected segments $(n=3)$. Cyst outline was smooth and cystobiliary communication noted in all cases. Deformation of cyst wall was noted in 2 cases. Isolated (left or right) IHBRD lateralised to the side of the cyst was most common $(n=4)$. CBD dilatation was seen in all cases with a mean calibre of $1.4 \mathrm{~cm}$. Concomitant extrabiliary rupture was seen in 3 cases. Endoscopic management was done in all cases without complications. Three patients underwent surgical excision after a mean period of 8 weeks following ERCP.

Conclusion: Isolated IHBRD lateralized to the side of hydatid cyst with CBD dilatation is the commonest ERCP finding of intrabiliary rupture of hydatid cyst.
\end{abstract}

KEYWORDS: ERCP, Hydatid cyst, intrabiliary rupture

\section{Introduction}

Biliary communication of hydatid cyst is a common occurrence at surgery. ${ }^{1}$ However, it is less often reported on imaging. ${ }^{2}$ This is due to the occult nature of most intrabiliary ruptures. ${ }^{3}$ These cases remain clinically silent. However, even these cases can progress to frank rupture presenting as a clinically significant syndrome comprising abdominal pain, fever and jaundice. ${ }^{4}$ Imaging evaluation of suspected intrabiliary rupture is based on both direct and indirect signs. ${ }^{5}$ Although the former are specific, they have a lower sensitivity. In most cases, one has to rely on a combination of indirect signs for detecting an intrabiliary rupture. Ultrasonography (USG), CT, MRI/ MRCP and ERCP are the imaging modalities utilised in this context. ERCP is the most sensitive and specific modality for the diagnosis of intrabiliary rupture. ${ }^{6}$ Owing to its invasive nature, 
CT and MRCP have been used with excellent results. ${ }^{7,8}$ However, ERCP has the advantage of biliary decompression and hence management of patients. ${ }^{9}{ }^{10}$ A combination of ERCP and a cross-sectional imaging technique represent the best method of evaluating patients with suspected intrabiliary rupture. ${ }^{8}$

\section{Material and methods}

A retrospective review of Radiology files from May 2012 to December 2013 was performed. We found six liver hydatid cyst cases in which a diagnosis of intrabiliary rupture was established based on CT $(n=1)$, MRI $(n=1)$ or ERCP $(n=4)$. USG reports were available for all the patients. Two cases in which CT/ MRI were performed were excluded. Thus we evaluated 4 cases. Diagnosis was based on typical ultrasound findings of internal membranes or daughter cysts in 3 patients, and cyst aspiration was performed in 1 patient. Diagnosis was based on the documentation of cystobilairy communication that was seen in all cases. Confirmation was based on surgical findings in 3 patients. All our patients were female with a mean age of 32 years (age range 22-43 years). ERCP examinations were performed on digital fluoroscopy unit (Shimadzu Digipro). Ultrasound findings recorded in each case included the number, location and size of cyst; cyst characteristics including the cyst outline whether smooth or irregular, cyst contents: hyperechoicor anechoic, deformation of cyst wall, intrahepatic biliary radicle dilatation: bilateral, right or left; $\mathrm{CBD}$ dilatation (calibre); presence of echogenic contents within CBD; evidence of extrabiliary rupture. ERCP evaluation included IHBRD, CBD dilatation, communication between cysts and intrahepatic biliary radicles (IHBR) and filling defects within the IHBR and CBD. Simultaneous biochemical or serological (hydatid serology) data were not available. Treatment details were accessible in all cases. However, long term follow up was not available.

\section{Results}

All patients except one had a single hydatid cyst. Cysts were located in the left lobe in all patients. Besides, in one patient, a cyst was located in the right lobe as well. Most common segments involved were segment II $(n=3)$ and III $(n=3)$. The mean cyst size was $6.2 \mathrm{~cm}$ (range $4-10 \mathrm{~cm}$ ). The cyst outline was smooth in all patients . Deformation of cyst wall was seen in 2 cases. Cystobiliary communication was seen in all cases.
Detached membranes were seen in 3 cysts. IHBRD was present in all cases. Isolated IHBRD (either right or left) was most common $(n=4)$. The isolated dilatation was lateralised to the side where the cyst was located. In one case, there was bilateral central and left peripheral dilatation. In another case, there was bilateral central and peripheral dilatation. CBD dilatation was seen in all cases. In all cases, dilatation was between 1-2 cm (mean $1.4 \mathrm{~cm}$, range 1.1-2.1 cm). In all cases, there was smooth distal tapering. Filling defects within CBD were seen in 3 cases (Figure 1). Concomitant extrabiliary rupture was seen in 3 cases.

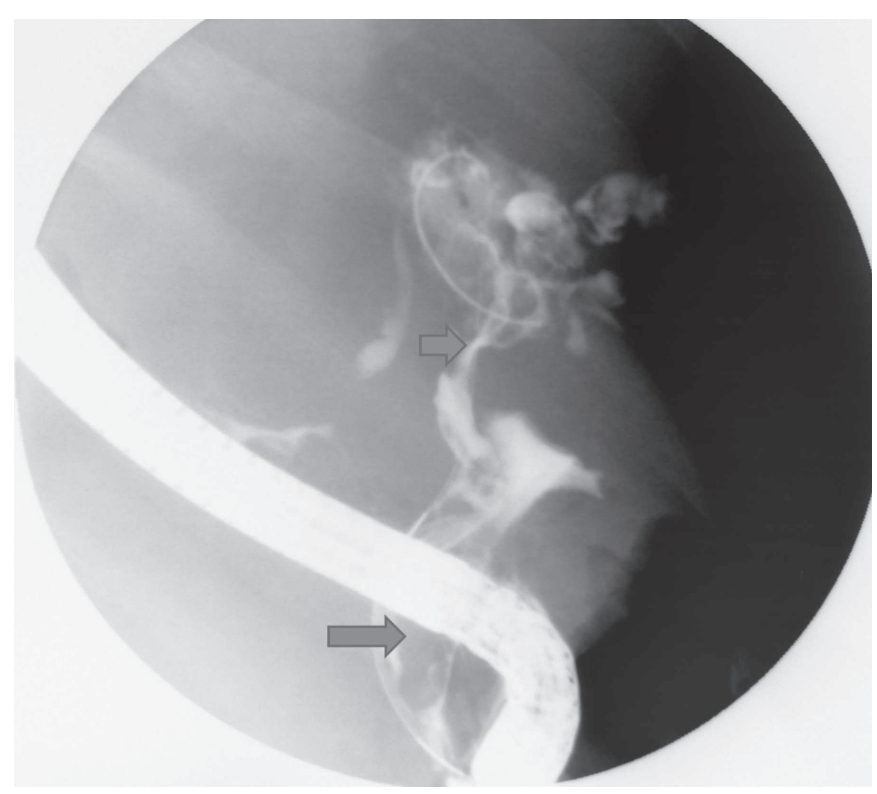

Figure 1: ERCP image shows isolated dilatation of the right ductal system (short arrow) with cystobiliary communication. Multiple filling defects are seen within the cyst and the CBD (arrow) which is dilated.

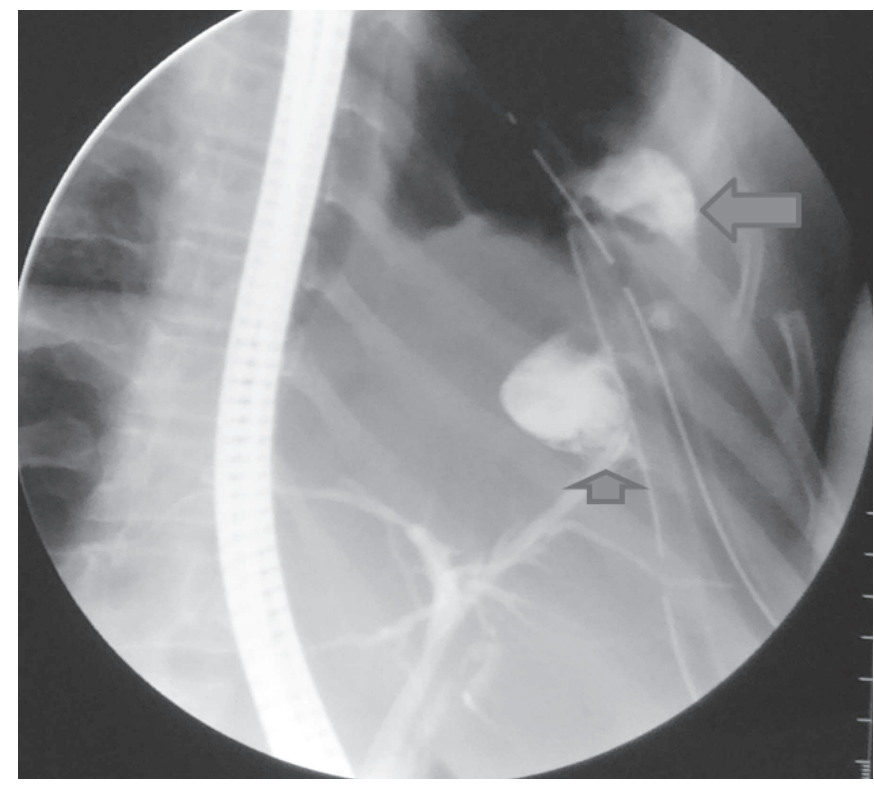

Figure 2: ERCP image demonstrates cystobiliary communication (short arrow) with intrapleural rupture (arrow). 
In two patients, there was pleural rupture (Figure 2). In the third case, there was subcapsular rupture along the right lobe of liver. All patients underwent endoscopic treatment in the form of endoscopic papillotomy and cyst material extraction. Surgery was performed in 3 patients after a mean period of 8 weeks from the time of acute presentation.

\section{Discussion}

Rupture into the bile ducts is one of the most common complications of hydatid cyst of liver. ${ }^{8}$ This is classified as a communicating cyst rupture; the other two forms are contained and direct rupture. ${ }^{11}$ Hydatid cyst rupture may be precipitated by physical trauma or excessive effort as in violent coughing or vomiting. ${ }^{12}$ Depending on the clinical presentation, the intrabiliary rupture is classified into frank or occult. ${ }^{3}$ Patients are acutely ill in the former, presenting with fever, abdominal pain and jaundice. ${ }^{4}$ It has an incidence between 3 and $17 \% .{ }^{12}$ In occult rupture, the only complaint may be abdominal pain. Signs are limited in this form of rupture. Imaging plays an important role in preoperative diagnosis of intrabiliary rupture and allows appropriate management.

Imaging signs of biliary communication are classified into direct and indirect. ${ }^{5} \mathrm{~A}$ visible communication between the cyst and the biliary radicles is the only direct sign. ERCP is considered the reference standard and the most reliable imaging technique for preoperative evaluation of biliary communication. Goumas K et al evaluated 9 patients with suspected biliary rupture with ERCP. Eight (88.9\%) patients were found to have a cystobiliary communication ${ }^{13}$. Similarly, in the study by Galati G et al, cytsobiliary communication was detected in $80 \%$ patients $(\mathrm{n}=8){ }^{9}$ In the present study, we could demonstrate cystobiliary communication in all cases. This sign, however has a low sensitivity, around $33 \%$ on $\mathrm{CT} / \mathrm{MRI}^{8}$. Indirect signs are often useful in making a diagnosis of intrabiliary rupture. Ultrasonography is often the initial imaging modality and allows evaluation of cyst characteristics and indirect signs of rupture. However, invariably it needs to be supplemented with additional imaging techniques. Deformation of cyst in the absence of treatment is an important sign of cyst rupture. ${ }^{8}$ We found this in two patients. Wani et al found cyst wall irregularity in 3 out of 6 patients on MDCT.7 Like cyst wall collapse, reduced intracystic pressure results in detachment of membranes seen as layered structures on USG/CT/ MRI and filling defects on $\mathrm{ERCP} / \mathrm{MRCP}$. We found evidence of detached membranes in 3 cases. Extrusion of cyst contents into the bile ducts results in filling defects in the IHBR and CBD. This finding was seen in 3 cases. Three patients (25\%) suffered filling defects within CBD in a study by Erden et al. ${ }^{8}$ The passage of cyst contents into the biliary tree also results in IHBRD. This is a common finding reported in all patients in the study by Wani et $\mathrm{al}^{7}$ and $75 \%$ patients in the study by Erden et al. ${ }^{8}$ We also noted IHBRD in all our patients. Though sensitive, these findings can also be seen in the presence of blood, pus or sludge. Beak-like projection was reported as an indirect sign of cyst rupture in the study by Erden et al. ${ }^{8}$ We did not evaluate this sign. In patients with biliary communication, other forms of rupture may also be evident. We saw this in $50 \%$ of our cases. In two cases, there was pleural rupture and in the third case, there was subcapsular rupture. Wani et al reported pleural $(\mathrm{n}=1)$ and subcapsular rupture $(\mathrm{n}=1)$ in their MDCT evaluation of 6 cases. $^{7}$

Besides detection of communication, ERCP plays an important role in the management of these patients by decompressing the biliary tree. ${ }^{9,10}$ Galati et al reported the utility of ERCP in management of patients with cystobiliary fistula. ${ }^{9}$ In this study of 10 patients with suspected biliary rupture, endoscopic sphincterotomy was performed in 7 when the fluid contained daughter cysts or pus. In three patients, in whom the biliary content was fluid only, sphincterotomy was not performed. All patients underwent surgical resection. It was concluded that after the introduction of selective preoperative ERC, the incidence of postoperative fistula significantly decreased. Singh et al also demonstrated the therapeutic role of ERCP in 7 patients with intrabiliary rupture of hydatid cyst. ${ }^{10}$ Seven patients underwent endoscopic treatment comprising endoscopic sphincterotomy, cyst material extraction and hypertonic saline lavage via nasobiliary catheter. Median follow up over 3.5 years documented cure in all cases. There were no treatment related complications. In the study by Goumas K et al, endoscopic management was successful in all patients $(n=9)$. Except for one patient with pulmonary hydatid cyst rupture during ERCP, no serious endoscopy-related complications were recorded. Similar to the results of previous studies, endoscopic management was effective in all cases and no major complications were encountered. However, ERCP is invasive and is being replaced by MRCP when diagnosis is the primary concern. ${ }^{8}$

In conclusion, ERCP allows the diagnosis of rupture of hepatic hydatid cysts with high sensitivity and specificity and allows relatively safe and effective endoscopic management. 


\section{References}

1. Langer IC, Rose DB, Keystone JS, Taylor BR, Langer B. Diagnosis and management of hydatid disease of the liver. Ann Surg. 1984:199:412-7.

2. Zargar SA ${ }^{1}$, Khuroo MS, Khan BA, Dar MY, Alai MS, Koul P. Intrabiliary mpture of hepatic hydatid cyst: sonographic and cholangiographic appearances. Gastrointest Radiol. 1992;17:41-5.

3. Mendez Montero JV, Arrazola Garcia J, Lopez Lafuente JL, Antela Lopez J, Mendez Fernandez R, Saiz Ayala A. Fat-fluid level in hepatic hydatid cyst: a new sign of rupture into the biliary tree? AJR Am J Roengentol. 1996;167:91-4.

4. Demircan O, Baymus M, Seydaoglu G, Akinoglu A, Sakman G. Occult cystobiliary communication presenting as postoperative biliary leakage after hydatid liver surgery: are there significant preoperative clinical predictors? Can J Surg. 2006,49:177-84.

5. Marti-Bonmati L, Menor Serrano F. Complications of hepatic hydatid cysts: ultrasound, computed tomography and magnetic resonance diagnosis. Gastrointest Radiol. 1990:15:119-25.

6. Magistrelli P, Masetti R, Coppola R, Costamagna G, Durastante $\mathrm{V}$, Nuzzo G, et al. Value of ERCP in the diagnosis and management of pre- and postoperative biliary complications in hydatid disease of the liver. Gastrointest Radiol. 1989;14:315-20.

7. Wani NA, Kosar T, Gojwari T, Robbani I, Choh NA, Shah AI, et al. Intrabiliary rupture of hepatic hydatid cyst: multidetector- row CT demonstration. Abdom Imaging. 2011;36:433-7.

8. Erden A, Ormeci N, Fitoz S, Erden I, Tanju S, Genç Y. Intrabiliary Rupture of Hepatic Hydatid Cysts: Diagnostic Accuracy of MR Cholangiopancreatography. AJR Am J Roengentol. 2007;189:W84-9.

9. Galati G, Sterpetti AV, Caputo M, Adduci M, Lucandri G, Brozzetti S, et al. Endoscopic retrograde cholangiography for intrabiliary rupture of hydatid cyst. Am J Surg. 2006;191:206-10.

10. Singh V, Reddy DC, Verma GR, Singh G. Endoscopic management of intrabiliary-ruptured hepatic hydatid cyst. Liver Int. 2006;26:621-4.

11. Spârchez Z $Z^{1}$, Osian G, Onica A, Bãrbântã C, Tanpãu M, Pascu O. Ruptured hydatid cyst of the liver with biliary obstruction: presentation of a case and review of the literature. Rom $J$ Gastroenterol 2004:13;245-50.

12 Avcu S, Unal O, Arslan H. Intrabiliary rupture of liver hydatid cyst: a case report and review of the literature. Cases $J$ 2009;2:6455.

13 Goumas K, Poulou A, Dandakis D et al. Role of endoscopic intervention in biliary complications of hepatic hydatid cyst disease. Scand $J$ Gastroenterol 2007;42:1113-9.

14 Kapoor A, Vashisht S, Sharma R. Intrabiliary rupture of hepatic hydatid cyst-imaging features. Trop Gastroenterol 1998;19:115-7. 